\title{
Statistical analysis of laser driven protons using a high-repetition-rate tape drive target system
}

\author{
Muhammad Noaman-ul-Haq, ${ }^{1,2, *}$ Hamad Ahmed, ${ }^{3}$ Thomas Sokollik, ${ }^{1,2, \dagger}$ Lule Yu, ${ }^{1,2}$ \\ Zezhou Liu, ${ }^{1,2}$ Xiaohui Yuan, ${ }^{1,2}$ Fang Yuan, ${ }^{1,2}$ Mohammad Mirzaie, ${ }^{1,2}$ Xulei Ge, ${ }^{1,2,4}$ \\ Liming Chen, ${ }^{1,2,5}$ and Jie Zhang ${ }^{1,2}$ \\ ${ }^{1}$ Key Laboratory for Laser Plasmas (Ministry of Education) \\ and Department of Physics and Astronomy, Shanghai Jiao Tong University, Shanghai 200240, China \\ ${ }^{2}$ Collaborative Innovation Center of IFSA (CICIFSA), Shanghai Jiao Tong University, \\ Shanghai 200240, China \\ ${ }^{3}$ School of Mathematics and Physics, Queen's University Belfast, Belfast BT71NN, United Kingdom \\ ${ }^{4}$ State Key Laboratory of Surface Physics and Department of Physics, Fudan University, \\ Shanghai 200433, China \\ ${ }^{5}$ Beijing National Laboratory of Condensed Matter Physics, Institute of Physics, \\ Chinese Academy of Sciences, Beijing 100190, China \\ (Received 5 December 2016; published 14 April 2017)
}

\begin{abstract}
One of the challenges for laser-driven proton beams for many potential applications is their stability and reproducibility. We investigate the stability of the laser driven proton beams through statistical analysis of the data obtained by employing a high repetition rate tape driven target system. The characterization of the target system shows the positioning of the target within $\sim 15 \mu \mathrm{m}$ in the focal plane of an off-axis parabola, with less than a micron variation in surface flatness. By employing this stable target system, we study the stability of the proton beams driven by ultrashort and intense laser pulses. Protons with maximum energies of $\sim 6 \pm 0.3 \mathrm{MeV}$ were accelerated for a large number of laser shots taken at a rate of $0.2 \mathrm{~Hz}$ with a stability of less than 5\% variations in cutoff energy. The development of high repetition rate target system may provide a platform to understand the dynamics of laser driven proton beams at the rate required for future applications.
\end{abstract}

DOI: 10.1103/PhysRevAccelBeams.20.041301

\section{INTRODUCTION}

Current state of the art high power laser systems have the capability to generate ultrashort laser pulses at high repetition rate required for many applications [1-3], for instance, acceleration of particles (electrons and ions) using high power lasers. Acceleration of ions in the interaction of intense and ultrashort laser pulses with solid targets [4] do not allow to explore the full capability of the laser systems viz. high repetition rate, mainly due to the complications in replacing targets at such a high rate. So far, most of the investigations have been made in a single shot mode [4,5], yet require a systematic study at high repetition rate to investigate the stability and reproducibility of the ion beams. For potential applications of the laser driven ion beams, such as ion implantation of semiconductors, high energy physics, production of radio-isotopes and medical applications [4,5],

\footnotetext{
*haq.noaman@sjtu.edu.cn

†ThomasSokollik@yahoo.de
}

Published by the American Physical Society under the terms of the Creative Commons Attribution 4.0 International license. Further distribution of this work must maintain attribution to the author(s) and the published article's title, journal citation, and DOI. repetition rates of greater than $10 \mathrm{~Hz}$ would ideally be required. In addition to study the stability of the ion beams with a high repetition rate target system, closed loop optimization of various laser diagnostics would be possible [6,7].

In addition to the ongoing progress in laser technology, development of compatible high-repetition rate target systems is essential. In this context, there are only a few target systems available, like droplet systems [8], gas jets [9], and tape targets [10]. Tape driven targets can provide continuous and fresh supply at high repetition rate without extra efforts on vacuum systems required for droplets targets and gas jets [10-12]. Moreover, it facilitates to investigate the stability of the laser driven ion beams in the light of developing next generation particle accelerators for the aforementioned applications.

In this paper, we present a low-cost and high repetition rate VHS (Video Home System) tape drive target system [13] for stable and reproducible acceleration of protons in the interaction with intense laser pulses. High performance and vacuum compatible DC motors with a feedback loop for current optimization make this system viable for laser driven protons experiments. By employing the tape drive target system, a large number of shots were taken at a rate of $0.2 \mathrm{~Hz}$. The data suggests that $\sim 6 \mathrm{MeV}$ protons were accelerated in the interaction with a fluctuation of $0.26 \mathrm{MeV}$ 
in the maximum proton energy. Furthermore, a correlation analysis of the proton beam parameters exhibits a correlation of maximum proton energy and flux to laser energy fluctuations. This target system can be used to verify, in addition, to investigate angular properties of the proton beams and source fluctuations, existing scaling laws and predications of theoretical models [14-17] at high repetition rate.

\section{TAPE DRIVE TARGET SYSTEM AND ITS CHARACTERIZATIONS}

The tape drive target system developed for this study is illustrated in Fig. 1(a). It is based on two motorized spools for winding and unwinding of $\sim 13 \mathrm{~mm}$ wide and more than $100 \mathrm{~m}$ long thin VHS tape of $15 \mu \mathrm{m}$ thickness. In order to hold the tape between the two spools four highly polished stainless steel (SS) bolts were used. The wedge shape on the structure plate was designed for unobstructed movement of ions towards the detector. The design is simple but versatile as the base plate is adjustable and can be fixed on the upper or lower part of the structure plate. Therefore, the system can easily be installed in different experimental setups. High performance and vacuum compatible DC motors (Maxon Motor) with a computer control system ensured the precise movement of the tape target. In comparison to the tape drive system described in Ref. [11] that can only be operated in low vacuum $\left(5 \times 10^{-2} \mathrm{mbar}\right)$ due to noncompatible motors and clutches for high vacuum, our tape drive system is compatible with high vacuum $\left(10^{-5} \mathrm{mbar}\right)$, typically required in experiments using ultrashort laser pulses. A rotary encoder was installed to measure the position, speed, and acceleration of the tape. This information was utilized in the feedback loop that controls the current to the motors, which allows a smooth movement of the tape. Figure 1(b) shows the schematic of the control system for the tape drive system.
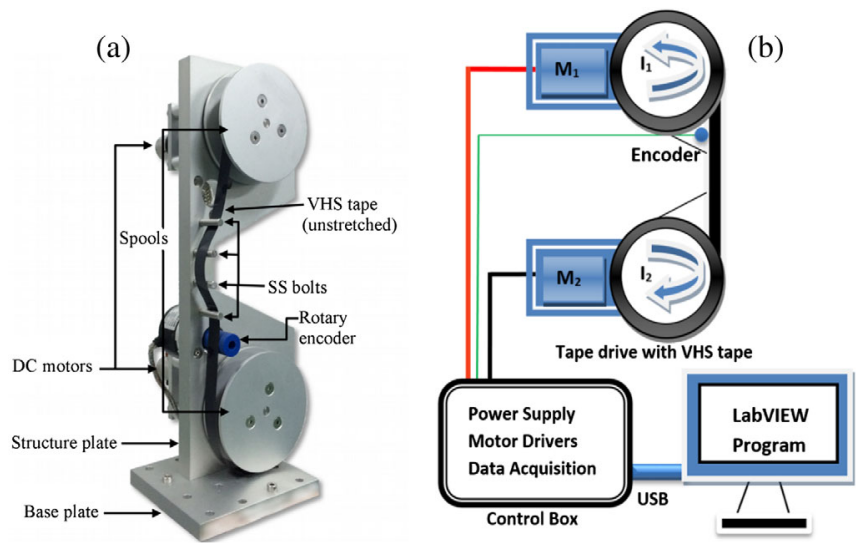

FIG. 1. (a) Photograph of the tape drive target system. (b) Schematic of the control system for the tape drive target where $M_{1}$ and $M_{2}$ represents stepper motors with $I_{1}$ and $I_{2}$ are the corresponding currents.
The VHS tapes used in this study were low-cost and commercially available. The surface roughness of these tapes were found less, having $14.3 \mathrm{~nm}$ (RMS) and $168.2 \mathrm{~nm}$ peak to valley roughness, as compared to other tapes e.g. Kapton tape [18]. These types of VHS tapes (iron-oxide particles with Mylar as a carrier substrate) have already been used for generating higher order harmonics and as a plasma mirror [13,19-21]. Having combination with plastic (Mylar) they have good mechanical strength, moreover, reduce the generation of electromagnetic pulses during ion acceleration, typically observed in the case of metal targets which interrupt operation of electronic devices [22,23]. Although for such tape targets debris management is an important issue to protect the optical components, it can be overcome by using thin glass pellicle or thin Mylar sheet [24]. In order to observe the stability of tape target positioning, we used an imaging system consists of a 10 bit Firefly CCD camera (Aptina MT9V022) with a microscope objective of 10x magnification. This allows us to monitor the fluctuations of the thin side of the VHS tape in the laser focus direction during its movements. Thus for repetitive runs of the tape on the drive system the fluctuations in position were observed from the mean position. The histogram for these observations is plotted in Fig. 2 and shows the surface position reproducibility and stability of the tape target. It is important to mention here that the fluctuation in surface positioning of the tape target in focal plane of off-axis parabola (OAP) was $\sim 14 \mu \mathrm{m}$, which an order of magnitude less than the position reproducibility reported in the Refs. [11,25], where the target position was reproduced in $\sim 100 \mu \mathrm{m}$ which is the limit of Rayleigh range of OAP, resulting in large variation of laser intensity. Considering the fluctuations, it can be inferred from Fig. 2 that $95 \%$ of the laser shots would be within $28 \mu \mathrm{m}$.

Another important requirement for such tape targets is the flatness of the targeted area, i.e., free from the ripples

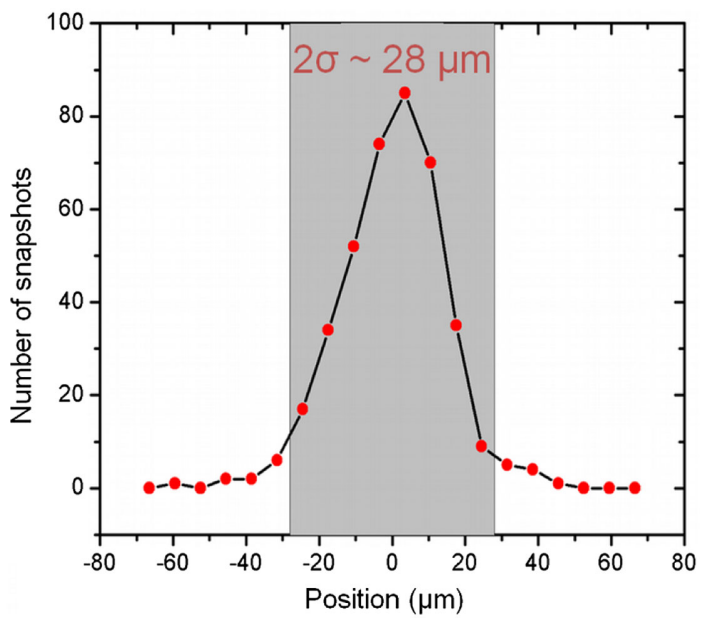

FIG. 2. Surface position reproducibility of the moving tape along the normal to the surface by imaging the thin side of the tape using CCD camera. 

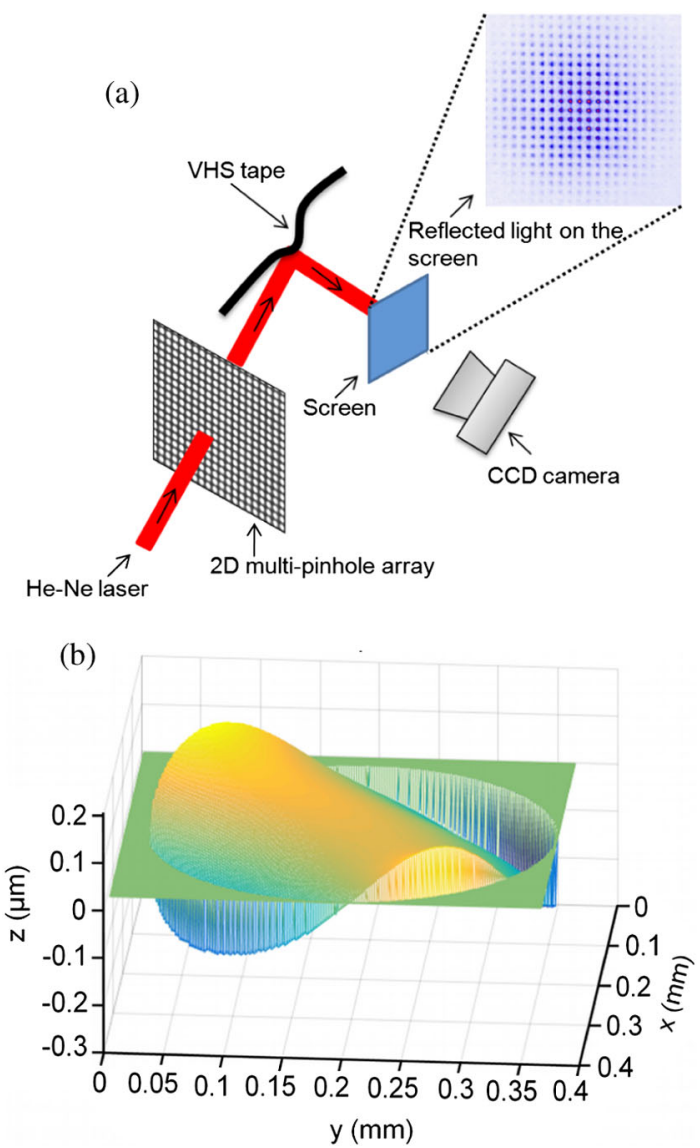

FIG. 3. Targeted area surface monitoring. (a) Schematic of the setup consisting of He-Ne laser with a multipinhole array and a CCD camera, (b) 3D plot of the reconstructed surface of the targeted area using Zernike polynomials.

[13]. We used a low intensity $\mathrm{He}-\mathrm{Ne}$ laser and a multipinhole array of $\sim 165 \mu$ m diameter and $\sim 220 \mu \mathrm{m}$ interhole spacing to study the wave front of the reflected light from the targeted area as shown in Fig. 3(a). By using a charged coupled device (CCD) camera, we detected the reflected beam and measured the deviation from a regular reference pattern. The surface of the target area was reconstructed using Zernike polynomials [12,26] and is shown in Fig. 3(b). The fluctuations in the probed surface area were found less than $1 \mu \mathrm{m}$ as the tape was driven with the optimum current of $0.2 \mathrm{~A}$ in the stepper motors.

\section{EXPERIMENT}

The experiment was performed at the Laboratory for Laser Plasmas (LLP), Shanghai Jiao Tong University. Ti: Sapphire based laser system was used which can deliver p-polarized pluses of $\sim 25 \mathrm{fs}$ long and energy $\sim 5 \mathrm{~J}$. While in the experiment presented here, the laser pulse energy was $\sim 1-2 \mathrm{~J}$. The repetition rate of the laser system is $10 \mathrm{~Hz}$, however, the experiment discussed here, was conducted at $0.2 \mathrm{~Hz}$. In addition to compatible with the target system, this allows sufficient time for effective cooling of the lasing materials, which leads to the stability of the laser beam

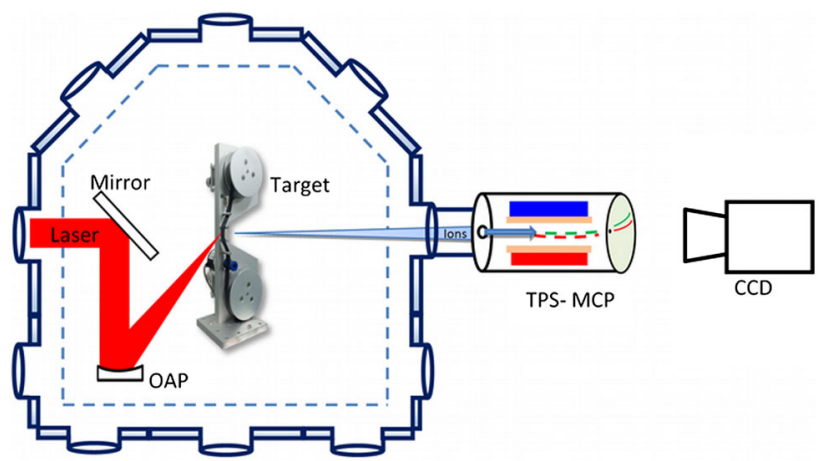

FIG. 4. Schematic of the experimental setup for acceleration for ions in the interaction of intense laser pulses with VHS tape drive target system.

parameters and focusability [27]. The amplified spontaneous emission contrast (ASE) to the main peak was measured to be $10^{-8}$ at $10 \mathrm{ps}$ by a scanning third-order auto correlator [27]. Figure 4 shows the schematic of the experimental setup. Laser pulses were focused to $\sim 6 \mu \mathrm{m}$ spot (FWHM), using a $f / 4$ off-axis parabolic mirror, containing $25 \%$ of energy in FWHM. The resulting maximum intensity on the target was $\sim 6 \times 10^{19} \mathrm{~W} / \mathrm{cm}^{2}$. The tape drive system with VHS tape of $15 \mu \mathrm{m}$ was mounted on a motorized three dimensional (3D) translation stage system at the center of the interaction chamber. A Thomson parabola spectrometer (TPS) was installed at normal to the target rear surface in a separate vacuum chamber, which was pumped down to $10^{-6}$ mbar pressure. A pinhole of $100 \mu \mathrm{m}$ diameter was installed at a distance of $\sim 755 \mathrm{~mm}$ from the target results in a solid angle of $\sim 1.4 \times 10^{-8} \mathrm{sr}$. The ions traces were collected on a phosphor screen of a microchannel plate (MCP) detector of $40 \mathrm{~mm}$ diameter with 14-bit CCD camera (Point Grey).

\section{RESULTS AND DISCUSSION}

In the interaction of ultrashort intense laser with solid targets, ions are accelerated mainly by well established target normal sheath acceleration (TNSA) [4], where the ions acceleration is because of large sheath electric field $(\mathrm{TV} / \mathrm{m})$ on the rear side of the target, generated by hot electrons. Since protons are lighter than other hydrocarbon containments present on the target rear surface, they are accelerated most effectively in the direction normal to the target [4]. The spectrum of the accelerated protons is typically broadband with a sharp cutoff in the energy. Since the cutoff energy and flux of the protons beams are important features of the accelerated protons [4], the stability and constancy of these features are important for the many future applications.

In order to investigate the stability of TNSA driven proton beams, we performed the experiment employing the VHS tape drive target system at the repetition rate of $0.2 \mathrm{~Hz}$. The energy spectrum of the protons is shown in Fig. 5(a). 

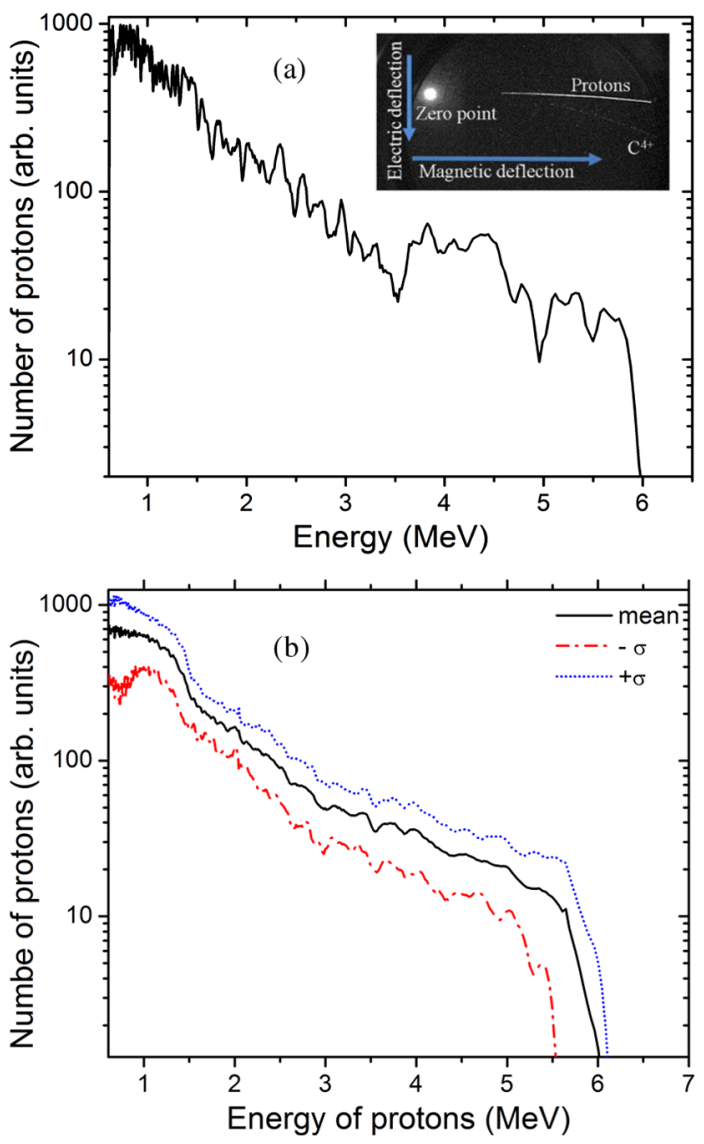

FIG. 5. Energy spectra of the accelerated protons. (a) Typical proton spectrum extracted from the raw images, shown in the inset, on the MCP detector, (b) Mean proton energy spectrum for 50 consecutive laser shots.

The raw image in the inset shows ions traces and a bright spot, typically known as zero point, due to undeflected particles and radiations. A LabVIEW [28] based program was developed to extract the protons energy spectrum. Figure 5(b) shows an average proton spectrum for fifty consecutive laser shots. The mean proton cutoff energy is $5.85 \mathrm{MeV}$. The relative proton number variations are shown by standard deviation $( \pm \sigma)$ line bounds. The spectrum shows slightly higher variation for low energy $<1.5 \mathrm{MeV}$, which can be due to the variations in interaction conditions, e.g. ASE pedestal variation from shot to shot, which leads to different pre-plasma conditions resulting in changes in proton flux and cutoff energy [29,30]. Moreover, the difference in plasma condition may lead to two protons source, essentially with different electron temperatures, that may result in modulated proton spectrum [31].

Table I highlights the statistical analysis of the variations in the proton beam parameters for fifty consecutive shots. The variations are within $\sim 1 \mathrm{MeV}$ between maximum and minimum proton cutoff energy with $0.26 \mathrm{MeV}$ standard deviation, which implies that the cutoff energy of the proton beams is significantly stable with the coefficient of variation $(\mathrm{CV})$ about $4 \%$. The flux of the proton beam is crucial for applications including cancer therapy [32]. Variation in the proton flux is $\sim 35 \%$, which is higher than the variation in the proton cutoff energy. As mentioned earlier, it could be due to different pre-plasma conditions in different shots, which results in different plasma scale length and hence, the laser coupling to the target. Table I shows slightly higher fluctuations $(\sim 18 \%)$ in laser energy [33]. Reducing the laser energy fluctuations may result in relatively stable parameters of the proton beams.

In comparison to the results presented in the Refs. [11,25,34], where proton energies of $1 \mathrm{MeV}$, $1.2 \mathrm{MeV}$ and $1.5 \mathrm{MeV}$ respectively were measured in forward direction using tape targets, higher proton cutoff energy $\sim 6 \mathrm{MeV}$ is observed from $15 \mu \mathrm{m}$ thick low-cost VHS tape in the forward direction. Moreover, in the Ref. [35] the proton beams stability was measured for $7.5 \mu \mathrm{m}$ thick polyimide tape target for 13 and 40 consecutive shots at a rate of $1 \mathrm{~Hz}$, in different laser contrast conditions (without and with saturable absorber respectively). With different laser contrast conditions, maximum proton energies of $2.6 \mathrm{MeV}$ and $3.1 \mathrm{MeV}$ were observed with $23 \%$ and $6 \%$ fluctuations respectively in Ref. [35]. We obtained $\sim 6 \mathrm{MeV}$ protons with $4 \%$ fluctuations for 50 consecutive shots at $0.2 \mathrm{~Hz}$, with an order of magnitude less laser intensity as compared to Ref. [35].

In order to understand the correlation between proton beam parameters and laser energy variations in our data, we performed Pearson correlation analysis as shown in Fig. 6. Significant correlations are present among proton flux and proton maximum energy to the laser energy fluctuations. Figure 6(a) and (b) shows the corresponding coefficients and the p-values respectively. Proton flux has significant positive correlation and needs further investigation to understand the dependence on the laser energy. Similarly, a positive increasing trend is clear between proton cutoff energy and laser energy. Figure 6(c) shows the trend of laser energy variation and proton beam parameters.

TABLE I. Statistics of proton beam parameters.

\begin{tabular}{|c|c|c|c|c|c|}
\hline Parameters & Mean & SD & Variance & Minimum & Maximum \\
\hline Max. energy of protons $(\mathrm{MeV})$ & 5.85 & 0.26 & 0.07 & 5.32 & 6.31 \\
\hline Flux of protons (arb. units) & $2.24 \times 10^{5}$ & $8.0 \times 10^{4}$ & $6.42 \times 10^{5}$ & $1.08 \times 10^{5}$ & $4.43 \times 10^{5}$ \\
\hline Laser energy $(\mathrm{J})$ & 1.44 & 0.26 & 0.07 & 0.87 & 1.85 \\
\hline
\end{tabular}




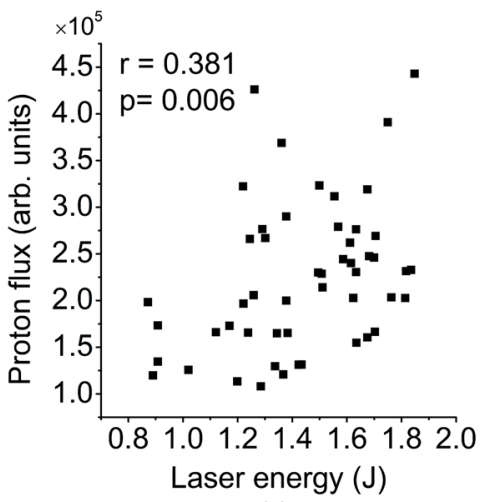

(a)

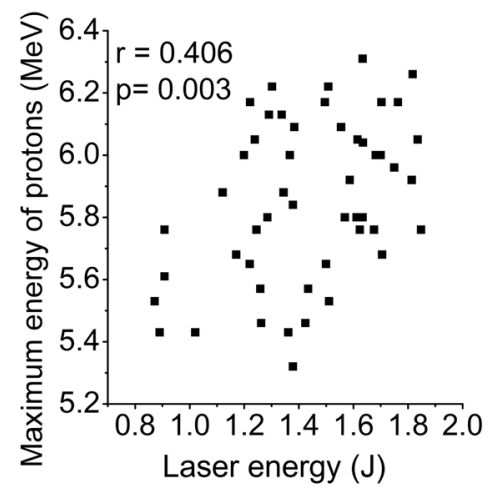

(b)
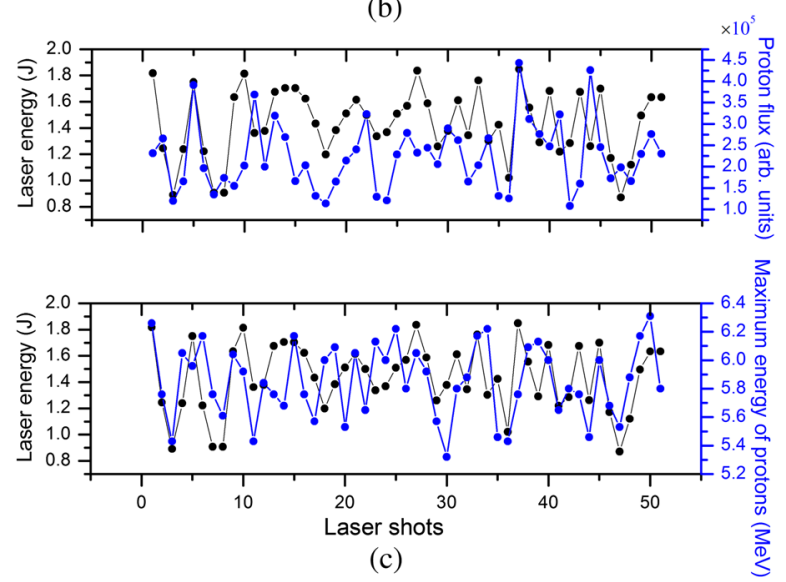

FIG. 6. The correlation scatter plots and Pearson correlation coefficient values of proton beam parameters versus laser energy. (a) proton flux, (b) protons maximum energy, and (c) trend of laser energy and proton beam parameters for fifty shots.

\section{SUMMARY}

In summary, we investigated the stability and reproducibility of the laser driven proton beams using a low-cost, high repetition rate VHS tape drive target system. The proton spectrum was found to be reproducible with only $4 \%$ fluctuations in maximum proton energy $(\sim 6 \mathrm{MeV})$. By taking numerous laser shots at relatively high repetition rate, we are able to do statistical analysis of the proton beam parameters. Significant correlation is found between maximum energy and the flux of the proton beams to laser energy fluctuations. Although a large number of shots were taken at a rate less than the capability of the laser system $(10 \mathrm{~Hz})$, it could provide a platform to understand the different characteristics of the proton beams viz. source size, beam divergence and spectrum at high repetition rate, which would be useful to distinguish different mechanisms involved in the acceleration of ions. For example, by employing higher repetition rate (1-5) $\mathrm{Hz}$ tape drive system with microns/sub-microns foils and high contrast, intense laser pulses, interplay between different acceleration mechanisms can be studied.

Data associated with research published in this article can be accessible at [36].

\section{ACKNOWLEDGMENTS}

This work was supported by the National Basic Research Program of China (under Grant No. 2013CBA01500), the National Natural Science Foundation of China (under Grants No. 11421064 and No. 11475113). Thomas Sokollik appreciates the support from Thousand Young Talents Program of China.

[1] S. Gales, Laser driven nuclear science and applications: The need of high efficiency, high power and high repetition rate laser beams, Eur. Phys. J. Spec. Top. 224, 2631 (2015).

[2] G. A. Mourou, T. Tajima, and S. V. Bulanov, Optics in the relativistic regime, Rev. Mod. Phys. 78, 309 (2006).

[3] ELI-Extreme Light Infrastructure Science and Technology with Ultra-Intense Lasers Whitebook, edited by G. A. Mourou, G. Korn, W. Sandner, and J. L. Collier (THOSS Media GmbH, Berlin, 2011).

[4] A. Macchi, M. Borghesi, and M. Passoni, Ion acceleration by superintense laser-plasma interaction, Rev. Mod. Phys. 85, 751 (2013).

[5] H. Daido, M. Nishiuchi, and A. S. Pirozhkov, Review of laser-driven ion sources and their applications, Rep. Prog. Phys. 75, 056401 (2012).

[6] Z.-H. He, B. Hou, V. Lebailly, J. A. Nees, K. Krushelnick, and A. G. R. Thomas, Coherent control of plasma dynamics, Nat. Commun. 6, 7156 (2015).

[7] Z.-H. He, B. Hou, G. Gao, V. Lebailly, J. A. Nees, R. Clarke, K. Krushelnick, and A. G. R. Thomas, Coherent control of plasma dynamics by feedback-optimized wavefront manipulationa), Phys. Plasmas 22, 056704 (2015).

[8] T. Sokollik, M. Schnürer, S. Steinke, P. V. Nickles, W. Sandner, M. Amin, T. Toncian, O. Willi, and A. A. Andreev, Directional Laser-Driven Ion Acceleration from Microspheres, Phys. Rev. Lett. 103, 135003 (2009).

[9] F. Sylla, M. Veltcheva, S. Kahaly, A. Flacco, and V. Malka, Development and characterization of very dense submillimetric gas jets for laser-plasma interaction, Rev. Sci. Instrum. 83, 033507 (2012).

[10] M. Nishiuchi et al., Focusing and spectral enhancement of a repetition-rated, laser-driven, divergent multi-mev proton beam using permanent quadrupole magnets, Appl. Phys. Lett. 94, 061107 (2009). 
[11] T. Nayuki, Y. Oishi, T. Fujii, K. Nemoto, T. Kayoiji, Y. Okano, Y. Hironaka, K. G. Nakamura, K. Kondo, and K. Ueda, Thin tape target driver for laser ion accelerator, Rev. Sci. Instrum. 74, 3293 (2003).

[12] T. Sokollik, Investigations of Field Dynamics in Laser Plasmas with Proton Imaging (Springer, Berlin, Heidelberg, 2011), p. 17.

[13] B. H. Shaw, S. Steinke, J. Van Tilborg, and W. P. Leemans, Reflectance characterization of tape-based plasma mirrors, Phys. Plasmas 23, 063118 (2016).

[14] S. C. Wilks and W. L. Kruer, Absorption of ultrashort, ultra-intense laser light by solids and overdense plasmas, IEEE J. Quantum Electron. 33, 1954 (1997).

[15] P. Mora, Plasma Expansion into a Vacuum, Phys. Rev. Lett. 90, 185002 (2003).

[16] J. Schreiber, F. Bell, F. Grüner, U. Schramm, M. Geissler, M. Schnürer, S. Ter-Avetisyan, B. M. Hegelich, J. Cobble, E. Brambrink, J. Fuchs, P. Audebert, and D. Habs, Analytical Model for Ion Acceleration by High-Intensity Laser Pulses, Phys. Rev. Lett. 97, 045005 (2006).

[17] M. Passoni and M. Lontano, Theory of Light-Ion Acceleration Driven by a Strong Charge Separation, Phys. Rev. Lett. 101, 115001 (2008).

[18] T. Sokollik, S. Shiraishi, J. Osterho, E. Evans, A. J. Gonsalves, K. Nakamura, J. van Tilborg, C. Lin, C. Toth, and W. P. Leemans, Tape-drive based plasma mirror, AIP Conf. Proc. 1299, 233 (2010).

[19] B. H. Shaw, J. van Tilborg, T. Sokollik, C. B. Schroeder, W. R. McKinney, N. A. Artemiev, V. V. Yashchuk, E. M. Gullikson, and W. P. Leemans, High-peak-power surface high-harmonic generation at extreme ultra-violet wavelengths from a tape, J. Appl. Phys. 114, 043106 (2013).

[20] J. van Tilborg, B. H. Shaw, T. Sokollik, S. Rykovanov, S. Monchocé, F. Quéré, Ph. Martin, A. Malvache, and W. P. Leemans, Spectral characterization of laser-driven solidbased high harmonics in the coherent wake emission regime, Opt. Lett. 38, 4026 (2013).

[21] S. Steinke, J. van Tilborg, C. Benedetti, C. G. R. Geddes, C. B. Schroeder, J. Daniels, K. K. Swanson, A. J. Gonsalves, K. Nakamura, N. H. Matlis, B. H. Shaw, E. Esarey, and W. P. Leemans, Multistage coupling of independent laser-plasma accelerators, Nature (London) 530, 190 (2016).

[22] Z.-Y. Chen, J.-F. Li, Y. Yu, J.-X. Wang, X.-Y. Li, Q.-X. Peng, and W.-J. Zhu, Influence of lateral target size on hot electron production and electromagnetic pulse emission from laser-irradiated metallic targets, Phys. Plasmas 19, 113116 (2012).

[23] M. De Marco, J. Krsa, J. Cikhardt, M. Pfeifer, E. Krousk, D. Margarone, H. Ahmed, M. Borghesi, S. Kar, L. Giuffrida, R. Vrana, A. Velyhan, J. Limpouch, G. Korn, S. Weber, L. Velardi, D. D. Side, V. Nassisi, and J. Ullschmied, Measurement of electromagnetic pulses generated during interactions of high power lasers with solid targets, J. Instrum. 11, C06004 (2016).

[24] S. J. Haney, K. W. Berger, G. D. Kubiak, P. D. Rockett, and J. Hunter, Prototype high-speed tape target transport for a laser plasma soft-x-ray projection lithography source, Appl. Opt. 32, 6934 (1993).

[25] T. Fujii, Y. Oishi, T. Nayuki, Y. Takizawa, K. Nemoto, T. Kayoiji, K. Horioka, Y. Okano, Y. Hironaka, K. G. Nakamura, and K. Kondo, Mev-order proton and carbon ion acceleration by irradiation of $60 \mathrm{fs}$ tw laser pulses on thin copper tape, Appl. Phys. Lett. 83, 1524 (2003).

[26] M. Schilke, J. Liebl, and C. Wnsche, Surface reconstruction by using zernike polynomials, Proc. SPIE Int. Soc. Opt. Eng. 10009, 1000910 (2016).

[27] Y. Fang, T. Yu, X. Ge, S. Yang, W. Wei, T. Yuan, F. Liu, M. Chen, J. Liu, Y. Li, X. Yuan, Z. Sheng, and J. Zhang, Combined proton acceleration from foil targets by ultraintense short laser pulses, Plasma Phys. Controlled Fusion 58, 045025 (2016).

[28] http://www.ni.com/labview/.

[29] A. J. Mackinnon, M. Borghesi, S. Hatchett, M. H. Key, P. K. Patel, H. Campbell, A. Schiavi, R. Snavely, S. C. Wilks, and O. Willi, Effect of Plasma Scale Length on Multi-Mev Proton Production by Intense Laser Pulses, Phys. Rev. Lett. 86, 1769 (2001).

[30] M. Kaluza, J. Schreiber, M. I. K. Santala, G. D. Tsakiris, K. Eidmann, J. Meyer-ter Vehn, and K. J. Witte, Influence of the Laser Prepulse on Proton Acceleration in Thin-Foil Experiments, Phys. Rev. Lett. 93, 045003 (2004).

[31] A. J. Kemp and H. Ruhl, Multispecies ion acceleration off laser-irradiated water droplets, Phys. Plasmas 12, 033105 (2005).

[32] S. V. Bulanov and V. S. Khoroshkov, Feasibility of using laser ion accelerators in proton therapy, Plasma Phys. Rep. 28, 453 (2002).

[33] S. Fourmaux, S. Buffechoux, B. Albertazzi, D. Capelli, A. Lvy, S. Gnedyuk, L. Lecherbourg, P. Lassonde, S. Payeur, P. Antici, H. Ppin, R. S. Marjoribanks, J. Fuchs, and J. C. Kieffer, Investigation of laser-driven proton acceleration using ultra-short, ultra-intense laser pulses, Phys. Plasmas 20, 013110 (2013).

[34] I. Spencer, K. W. D. Ledingham, P. McKenna, T. McCanny, R. P. Singhal, P. S. Foster, D. Neely, A. J. Langley, E. J. Divall, C. J. Hooker, R. J. Clarke, P. A. Norreys, E. L. Clark, K. Krushelnick, and J. R. Davies, Experimental study of proton emission from 60-fs, 200-mj high-repetition-rate tabletop-laser pulses interacting with solid targets, Phys. Rev. E 67, 046402 (2003).

[35] A. S. Pirozhkov et al., Laser-driven proton acceleration and plasma diagnostics with J-KAREN laser, Proc. SPIE Int. Soc. Opt. Eng. 7354, 735414 (2009).

[36] DOI: 10.17034/4d188aa3-8032-496a-9930-69fc246c93b4 